\title{
Secagem de bananas prata e d'água por convecção forçada
}

\author{
Drying of banana prata and banana d’água by forced convection
}

\author{
Soraia Vilela BORGES ${ }^{1}$, Maurício Cordeiro MANCINI², Jefferson Luiz Gomes CORRÊA ${ }^{3 *}$, Julia LEITE²
}

\begin{abstract}
Resumo
Estudou-se a influência de variáveis como cultivar, formato (cilindro e disco), branqueamento e condições do ar aquecido (temperatura: 50 e $70{ }^{\circ} \mathrm{C}$ e velocidade: 0,14 e $0,42 \mathrm{~m} / \mathrm{s}$ ) sobre o comportamento de secagem convectiva de bananas com uso de modelagem matemática. As bananas foram desidratadas em secador de bandejas e pesada em intervalos pré-determinados. O modelo exponencial foi bem ajustado às curvas de secagem $\left(R^{2}: 0,98-0,99\right)$, mostrando que os fatores mais influentes sobre a taxa de secagem foram a temperatura, a velocidade do ar e o branqueamento. De acordo com as constantes cinéticas apresentadas pelo modelo recomenda-se a secagem de banana, em qualquer dos formatos estudados, nas seguintes condições: para banana-prata, uso de branqueamento e secagem a $50{ }^{\circ} \mathrm{C} / 0,42 \mathrm{~m} / \mathrm{s}$; e para banana-d’água, sem uso de branqueamento e secagem a $70{ }^{\circ} \mathrm{C} / 0,42 \mathrm{~m} / \mathrm{s}$.

Palavras-chave: frutas desidratadas; cinética de secagem; branqueamento.
\end{abstract}

\begin{abstract}
The influence of variables such as cultivar, shape (cylinder and disc), blanching, and heated air conditions (temperatures of 50 and $70{ }^{\circ} \mathrm{C}$ and velocities of 0.14 and $0.42 \mathrm{~m} / \mathrm{s}$ ) on convective drying behavior of bananas using mathematical modeling. The bananas were dehydrated in a tray dryer and were weighed in predetermined periods of time. The exponential model showed good agreement with the drying curves $\left(\mathrm{R}^{2}:\right.$ 0.98-0.99) indicating that the factors that influenced the drying rate the most were temperature, air velocity, and blanching. According to the kinetics constants obtained with the model, the drying of bananas is recommended, including all shapes investigated under the following conditions: banana prata, blanching and drying at $50{ }^{\circ} \mathrm{C} / 0.42 \mathrm{~m} / \mathrm{s}$; and banana d'água, no blanching and drying at $70{ }^{\circ} \mathrm{C} / 0.42 \mathrm{~m} / \mathrm{s}$.

Keywords: dehydrated fruits; drying kinetics; blanching.
\end{abstract}

\section{Introdução}

A banana (Musa ssp.) é uma fruta de grande importância econômica, tanto para o Brasil quanto para vários outros países. A produção anual brasileira é, em média, de sete milhões de toneladas (INSTITUTO..., 2007). Esta fruta de pequeno valor agregado e elevado valor nutritivo possui processo rápido de deterioração, o que torna a comercialização do fruto in natura bastante difícil após o amadurecimento. Neste sentido, a utilização da desidratação como forma de evitar estas perdas aparece como uma interessante alternativa econômica.

Nos últimos anos, nota-se, no Brasil, um crescimento na comercialização de frutas desidratadas (ou passa) em casas de produtos naturais e também sua utilização em barras nutritivas. Em relação à banana, as cultivares mais utilizadas têm sido nanica e prata, podendo outras variedades apresentar adequação para este processo (MOTA, 2005). Os produtos obtidos de bananas de cultivo orgânico, em Curitiba, têm sido exportados para uma empresa suíça, apresentando taxas internas de retorno de $94 \%$; os obtidos de cultivo convencional apresentaram taxas de retorno de $14 \%$ no mercado nacional (BITTENCOURT; QUEIROZ; NEBRA, 2004); apontando o cultivo orgânico como promissor.
Para ampliação deste agronegócio, é importante conhecer os parâmetros ótimos do processo e garantir a obtenção de frutas passas com qualidade. Estes parâmetros vão desde a escolha da cultivar mais adequada, pré-tratamentos aplicados à matéria-prima, até otimização das condições de secagem (QUEIROZ; NEBRA, 2001; DEMIREL; TURHAN, 2003; BOUDHRIOUA; GIAMPAOLI; BONAZZI, 2003; MOTA, 2005; FERNANDES et al., 2006; LEITE; MANCINI; BORGES, 2007; KATEKAWA; SILVA, 2007).

A cinética de secagem é um assunto vastamente coberto pela literatura para diferentes produtos e mostra que, dentre as variáveis mais importantes, estão temperatura e velocidade do ar de secagem (QUEIROZ; NEBRA, 2001; DEMIREL; TURHAN, 2003; KROKIDA et al., 2003; LAHSASNI et al. 2004; GIRALDO-ZUNIGA et al., 2006; CORRÊA et al., 2007; NAGLE et al., 2008; KAYA; AYDIN; DINCER, 2008).

O branqueamento, além de inativar enzimas, tem sido usado em vários trabalhos sobre secagem osmótica como etapa prévia, com o objetivo de tornar a estrutura celular do tecido

${ }^{1}$ Ciência dos Alimentos, Universidade Federal de Lavras - UFL, CEP 37200-000, Lavras - MG, Brasil

2 Departamento de Ciência e Tecnologia de Alimentos, Universidade Federal Rural do Rio de Janeiro - UFRRJ, Seropédica - RJ, Brasil

${ }_{3}^{3}$ Departamento de Ciência dos Alimentos, Universidade Federal de Lavras - UFL, CEP 37200-000, Lavras - MG, Brasil, E-mail: jefferson@dca.ufla.br

${ }^{*}$ A quem a correspondência deve ser enviada 
mais aberta e facilitar a transferência de massa (LEWICKI; PAWLAK, 2003). Entretanto, para alguns alimentos, conforme as condições de temperatura e velocidade do ar de secagem, este pré-tratamento pouco altera a taxa de secagem e pode causar sua diminuição (DANDAMRONGRAK; YOUNG; MASON, 2002; DEMIREL; TURHAN, 2003).

Efeitos da geometria do produto têm sido pouco estudados ou levados em consideração na modelagem da secagem; a ênfase sempre é dada à espessura do produto (MADAMBA; DRISCOL; BUCLE, 1996; QUEIROZ; NEBRA, 2001). De forma similar, o efeito de cultivares de diferentes frutas sobre a taxa de secagem também é pouco pesquisado. A maioria dos estudos se refere à banana-d'água ou nanica por serem cultivares mais baratas do que a banana-prata.

Baseado no exposto, este trabalho tem como objetivos avaliar o efeito das condições do ar de secagem, branqueamento e geometria sobre o comportamento da secagem de bananas cv. Prata e cv. D’água. Um modelo matemático exponencial foi utilizado para auxiliar nesta avaliação.

\section{Material e métodos}

\subsection{Matéria-prima}

As bananas foram adquiridas no comércio local, no estádio de maturação apropriado para a secagem (amarelas com poucas pintas negras, segundo TRAVAGLINI et al., 1993), apresentando um conteúdo médio de umidade de $3,5 \mathrm{~g} / \mathrm{g}$, base seca (b.s.) para banana-prata e 4,4 g/g (b.s.) para banana-dágua. Foram higienizadas com água potável e corrente, descascadas manualmente e cortadas no formato disco $(0,5 \mathrm{~cm}$ de espessura $\mathrm{e}$ diâmetro médio de $3,5 \mathrm{~cm}$ ) ou cilindro ( $10 \mathrm{~cm}$ de comprimento e $3,5 \mathrm{~cm}$ de diâmetro).

O tratamento térmico de branqueamento foi feito pela submersão das amostras em água fervente $\left(100^{\circ} \mathrm{C}\right)$ por 1 minuto, após experimentos preliminares com vários períodos de branqueamento nesta temperatura, utilizando-se como parâmetro um teste de inativação enzimática com solução de guaiacol à 1\% (INSTITUTO ADOLFO LUTZ, 1985).

\subsection{Secagem}

A secagem da banana por convecção forçada foi realizada em um mini-secador de bandejas, cujos detalhes sobre funcionamento foram descritos por Leite (2002) e também utilizado em Leite, Mancini e Borges (2007). Os fatores avaliados foram temperatura $\left(50\right.$ e $\left.70{ }^{\circ} \mathrm{C}\right)$ e velocidade do ar $(0,14$ e $0,42 \mathrm{~m} / \mathrm{s})$, branqueamento (com e sem branqueamento) e geometria (cilindro e disco). A faixa de temperatura utilizada foi escolhida com base em temperaturas usualmente utilizadas para produtos alimentícios (BOUDHRIOUA; GIAMPAOLI; BONAZZI, 2003; GIRALDO-ZUNIGA et al., 2006; CORRÊA et al., 2007, 2008) e a faixa de velocidades do ar, com a superior três vezes maior que a inferior, foi escolhida para que se testasse a convecção forçada no processo. O teor de umidade foi obtido segundo a AOAC (ASSOCIATION..., 2002), em intervalos de tempo pré-determinados.

\subsection{Modelo matemático}

As curvas de cinética de secagem foram ajustadas com auxílio de um modelo exponencial, o qual que relaciona a taxa teor de umidade/teor de umidade inicial e o tempo de secagem, adotado previamente por Alcina, Silva e Brasileiro (1997), Midilli, Kucuk e Yapar (2002), Cano-Chauca et al. (2004), Lahsasni et al. (2004) e Corrêa et al. (2008). Conforme pode ser observado naqueles trabalhos, modelos do tipo exponencial representam adequadamente o fenômeno da cinética de secagem, tanto física como estatisticamente (altos coeficientes de correlação).

Para desenvolvimento do modelo matemático aqui utilizado, considerou-se que (Equação 1):

$\frac{d Y s^{*}}{d t}=-k Y s^{*}$

em que (Equação 2):

$Y s^{*}=\frac{Y s}{Y s_{0}}$

com a condição inicial

$Y_{\mathrm{S}} *(0)=1$

Desta forma, a evolução de $Y_{s}$ com o tempo é dada pela Equação 3:

$Y s^{*}=k_{0} \exp \left(-k_{1} t\right)$

em que $\mathrm{k}_{0}$ corresponde à condição inicial e deve ser próximo da unidade.

Os dados ajustados pela Equação (3) foram avaliados quanto ao coeficiente de correlação $\left(\mathrm{r}^{2}\right)$ e erro padrão (SE), de acordo com Pimentel-Gomes (1999).

\section{Resultados e discussão}

A Tabela 1 mostra os coeficientes de ajuste do modelo utilizado para diferentes condições experimentais desta pesquisa. Foram observados altos coeficientes de correlação $\left(r^{2}\right)$ e baixos valores de erro padrão (SE), significando um bom ajuste aos dados experimentais. Verificou-se ainda coeficiente $\mathrm{k}_{0}$ próximo da unidade, o que condiz com o significado físico da umidade inicial.

Midilli, Kucuk e Yapar (2002) apresentaram uma comparação entre o modelo utilizado neste trabalho e outros modelos que consideram a umidade de equilíbrio. Aqueles autores obtiveram ótimos ajustes para cogumelo, pistache e pólen e concluíram que o modelo em questão é bastante conveniente para aplicações práticas, embora não haja um embasamento teórico que o justifique.

A constante cinética $\mathrm{k}_{1}$ indica o quão rápida é a secagem e verifica-se maior rapidez em condições de altas temperaturas e velocidade de ar, sendo estas relações melhor visualizadas e discutidas pelas Figuras apresentadas neste trabalho.

Observa-se pelas Figuras 1 a 4 que tanto a temperatura quanto a velocidade do ar são influentes na secagem das duas variedades de banana em ambos os formatos, acelerando ou retardando a secagem conforme aumento ou diminuição nos limites extremos de ambas variáveis, respectivamente. Entretanto 
em condições intermediárias $\left(70{ }^{\circ} \mathrm{C} / 0,14 \mathrm{~m} / \mathrm{s}\right.$ e $\left.50^{\circ} \mathrm{C} / 0,42 \mathrm{~m} / \mathrm{s}\right)$, a cinética não apresenta muita diferença para banana-d'água, ao passo que, para banana-prata, maiores taxas de secagem foram obtidas nas condições de maior temperatura para o formato disco e menor temperatura para o formato cilíndrico.

Valores altos de temperaturas e velocidade de secagem favorecem maior transferência de massa e calor pelo favorecimento de altos coeficientes de difusão, diminuindo o tempo necessário para atingir menores valores de teor de umidade. Estas tendências têm sido reportadas em vários trabalhos sobre cinética (QUEIROZ; NEBRA, 2001; DEMIREL; TURHAN, 2003; KROKIDA et al., 2003; LAHSASNI et al., 2004; GIRALDO-ZUNIGA et al., 2006; NAGLE et al., 2008; KAYA; AYDIN; DINCER, 2008).

Tabela 1. Resultados do modelo matemático aplicado a curvas de secagem da banana sob diferentes condições experimentais.

\begin{tabular}{|c|c|c|c|c|c|}
\hline Ensaio & Velocidade $[\mathrm{m} / \mathrm{s}]$ & $\mathrm{k}_{0}$ & $\mathrm{k}_{1} \times 10^{3}$ & $\mathrm{r}^{2}$ & SE \\
\hline \multicolumn{6}{|c|}{ Banana-prata sem branqueamento } \\
\hline \multirow[t]{2}{*}{ Disco $50^{\circ} \mathrm{C}$} & 0,14 & 0,9802 & 3,507 & 0,9956 & 0,00061 \\
\hline & 0,42 & 0,9568 & 5,261 & 0,9932 & 0,00178 \\
\hline \multirow[t]{2}{*}{ Disco $70^{\circ} \mathrm{C}$} & 0,14 & 1,0253 & 7,291 & 0,9812 & 0,00491 \\
\hline & 0,42 & 1,0235 & 9,989 & 0,9926 & 0,00196 \\
\hline \multirow[t]{2}{*}{ Cilindro $50^{\circ} \mathrm{C}$} & 0,14 & 0,9746 & 2,616 & 0,9922 & 0,00127 \\
\hline & 0,42 & 0,9441 & 4,382 & 0,9911 & 0,00221 \\
\hline \multirow[t]{2}{*}{ Cilindro $70^{\circ} \mathrm{C}$} & 0,14 & 0,9964 & 3,410 & 0,9978 & 0,00031 \\
\hline & 0,42 & 0,9892 & 9,077 & 0,9812 & 0,00055 \\
\hline \multicolumn{6}{|c|}{ Banana-prata com branqueamento } \\
\hline \multirow[t]{2}{*}{ Disco $50^{\circ} \mathrm{C}$} & 0,14 & 0,9512 & 3,126 & 0,9841 & 0,00290 \\
\hline & 0,42 & 0,9761 & 8,565 & 0,9961 & 0,00091 \\
\hline \multirow[t]{2}{*}{ Disco $70{ }^{\circ} \mathrm{C}$} & 0,14 & 0,9819 & 5,339 & 0,9981 & 0,00048 \\
\hline & 0,42 & 0,9615 & 10,379 & 0,9867 & 0,00287 \\
\hline \multirow[t]{2}{*}{ Cilindro $50^{\circ} \mathrm{C}$} & 0,14 & 0,9859 & 3,471 & 0,9990 & 0,00023 \\
\hline & 0,42 & 0,9331 & 7,496 & 0,9728 & 0,00466 \\
\hline \multirow[t]{2}{*}{ Cilindro $70{ }^{\circ} \mathrm{C}$} & 0,14 & 0,9740 & 6,122 & 0,9954 & 0,00106 \\
\hline & 0,42 & 0,9409 & 7,493 & 0,9783 & 0,00435 \\
\hline \multicolumn{6}{|c|}{ Banana-d'água sem branqueamento } \\
\hline \multirow[t]{2}{*}{ Disco $50^{\circ} \mathrm{C}$} & 0,14 & 0,9753 & 3,123 & 0,9812 & 0,00137 \\
\hline & 0,42 & 0,9283 & 4,875 & 0,9830 & 0,00399 \\
\hline \multirow[t]{2}{*}{ Disco $70^{\circ} \mathrm{C}$} & 0,14 & 0,9814 & 5,668 & 0,9973 & 0,00074 \\
\hline & 0,42 & 1,0006 & 7,592 & 0,9964 & 0,00114 \\
\hline \multirow[t]{2}{*}{ Cilindro $50^{\circ} \mathrm{C}$} & 0,14 & 0,9624 & 2,649 & 0,9946 & 0,00089 \\
\hline & 0,42 & 0,9990 & 5,744 & 0,9972 & 0,00019 \\
\hline \multirow[t]{2}{*}{ Cilindro $70^{\circ} \mathrm{C}$} & 0,14 & 0,9855 & 5,629 & 0,9971 & 0,00063 \\
\hline & 0,42 & 0,9495 & 7,262 & 0,9902 & 0,00274 \\
\hline \multicolumn{6}{|c|}{ Banana-d'água com branqueamento } \\
\hline \multirow[t]{2}{*}{ Disco $50^{\circ} \mathrm{C}$} & 0,14 & 1,0100 & 5,563 & 0,9962 & 0,00046 \\
\hline & 0,42 & 1,0095 & 8,214 & 0,9974 & 0,00038 \\
\hline \multirow[t]{2}{*}{ Disco $70^{\circ} \mathrm{C}$} & 0,14 & 1,0171 & 4,499 & 0,9932 & 0,00089 \\
\hline & 0,42 & 0,9746 & 5,711 & 0,9938 & 0,00092 \\
\hline \multirow[t]{2}{*}{ Cilindro $50^{\circ} \mathrm{C}$} & 0,14 & 0,9713 & 3,853 & 0,9871 & 0,00090 \\
\hline & 0,42 & 0,9607 & 4,554 & 0,9862 & 0,00196 \\
\hline \multirow[t]{2}{*}{ Cilindro $70^{\circ} \mathrm{C}$} & 0,14 & 0,9804 & 5,884 & 0,9936 & 0,00098 \\
\hline & 0,42 & 0,9564 & 8,906 & 0,9841 & 0,00272 \\
\hline
\end{tabular}

A influência do branqueamento como pré-tratamento na secagem de banana-prata e banana-d'água é retratada nas Figuras 5 a 12. Observa-se que o branqueamento conduz a maiores taxas de secagem, porém, sua influência é acentuada com o aumento da velocidade do ar e em menores temperaturas de secagem. A $70^{\circ} \mathrm{C}$, costuma ocorrer gelatinização do amido e a velocidade do ar tem pouco efeito ou nenhum efeito (DEMIREL; TURHAN, 2003). Com o branqueamento há a um aumento na permeabilidade do alimento (AREVALO-PINEDO; MURR, 2007). Esta permeabilidade, aliada ao aumento da velocidade do ar, deve facilitar a penetração do ar no alimento, facilitando as transferências de calor e massa. Porém, a mesma permeabilidade deve ser prejudicada com a gelatinização do amido e, desta forma, o branqueamento não é influente a $70^{\circ} \mathrm{C}$.

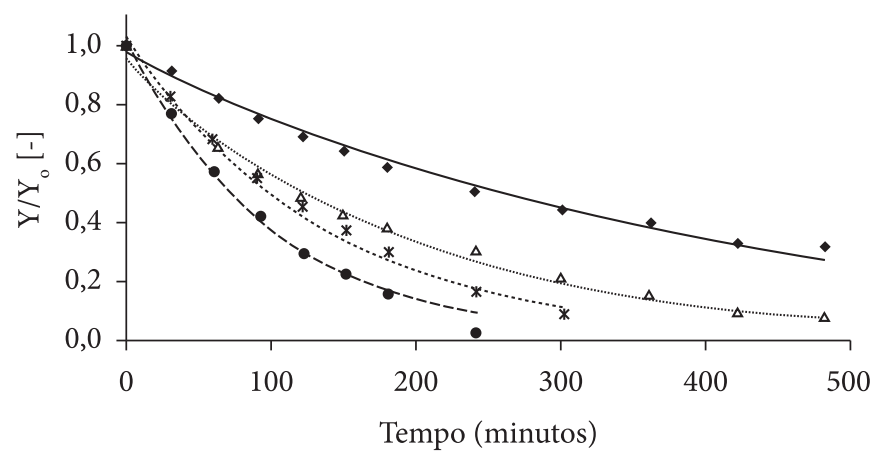

Figura 1. Curvas de secagem para banana cv. Prata no formato de disco em diferentes condições de secagem (exp: experimentais, sim: simulados).
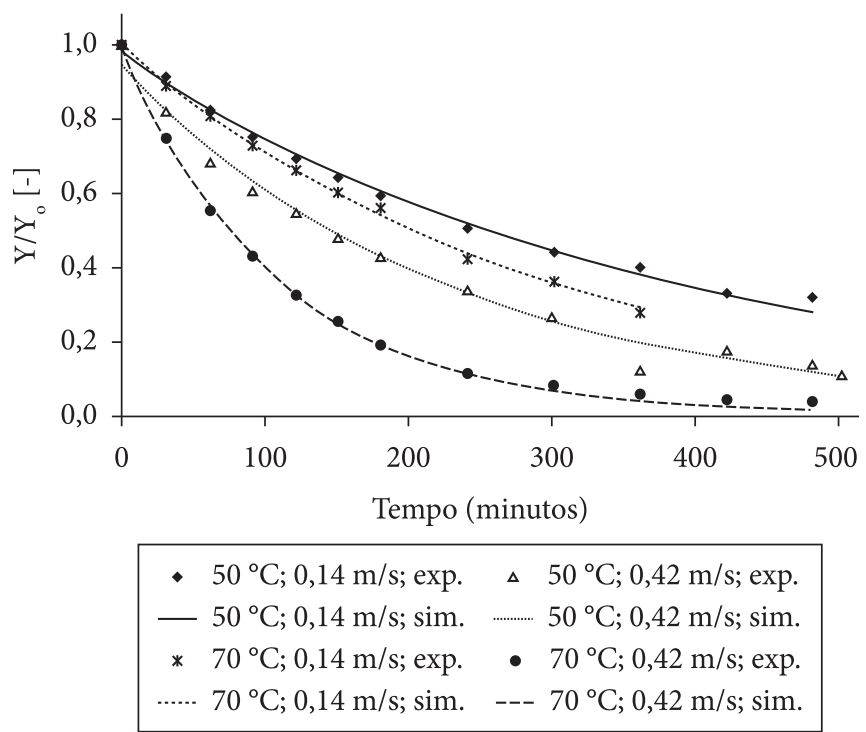

Figura 2. Curvas de secagem para banana cv. Prata no formato de cilindro em diferentes condições de secagem (exp: experimentais, sim: simulados). 


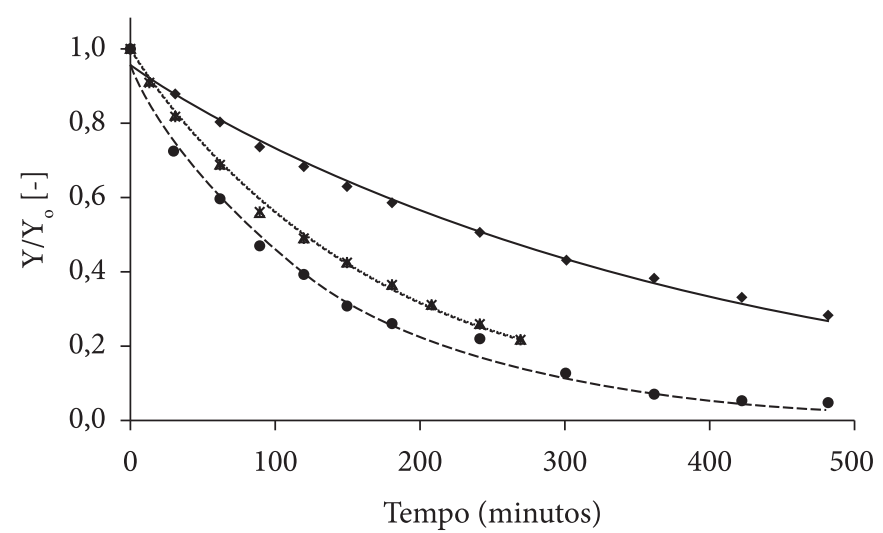

$$
\begin{aligned}
& \text { - } 50^{\circ} \mathrm{C} ; 0,14 \mathrm{~m} / \mathrm{s} ; \exp . \quad \Delta \quad 50^{\circ} \mathrm{C} ; 0,42 \mathrm{~m} / \mathrm{s} ; \exp \text {. } \\
& \text { — } 50^{\circ} \mathrm{C} ; 0,14 \mathrm{~m} / \mathrm{s} ; \operatorname{sim} \text {. ……. } 50^{\circ} \mathrm{C} ; 0,42 \mathrm{~m} / \mathrm{s} ; \operatorname{sim} \text {. } \\
& \text { * } 70^{\circ} \mathrm{C} ; 0,14 \mathrm{~m} / \mathrm{s} ; \exp \text {. } \quad 70^{\circ} \mathrm{C} ; 0,42 \mathrm{~m} / \mathrm{s} \text {; exp. } \\
& \text { …. } 70^{\circ} \mathrm{C} ; 0,14 \mathrm{~m} / \mathrm{s} ; \operatorname{sim} \text {. } \quad---70^{\circ} \mathrm{C} ; 0,42 \mathrm{~m} / \mathrm{s} ; \operatorname{sim} \text {. }
\end{aligned}
$$

Figura 3. Curvas de secagem para banana cv. D’água no formato de disco em diferentes condições de secagem (exp: experimentais, sim: simulados).

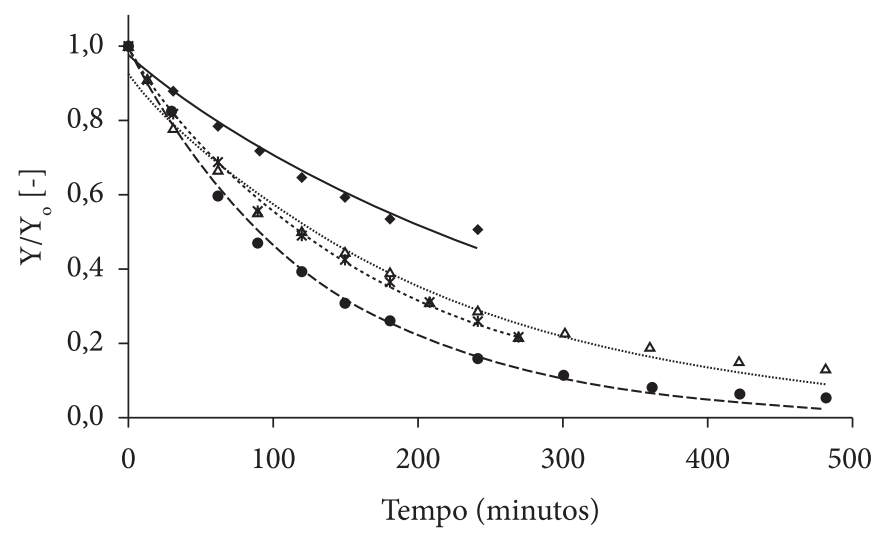

$$
\begin{aligned}
& \text { - } 50{ }^{\circ} \mathrm{C} ; 0,14 \mathrm{~m} / \mathrm{s} ; \exp . \quad \Delta \quad 50{ }^{\circ} \mathrm{C} ; 0,42 \mathrm{~m} / \mathrm{s} \text {; exp. } \\
& \text { — } 50{ }^{\circ} \mathrm{C} ; 0,14 \mathrm{~m} / \mathrm{s} ; \operatorname{sim} \text {. ……. } 50^{\circ} \mathrm{C} ; 0,42 \mathrm{~m} / \mathrm{s} ; \operatorname{sim} \text {. } \\
& \text { * } 70^{\circ} \mathrm{C} ; 0,14 \mathrm{~m} / \mathrm{s} \text {; exp. } \quad-70^{\circ} \mathrm{C} ; 0,42 \mathrm{~m} / \mathrm{s} \text {; exp. } \\
& \text { …. } 70{ }^{\circ} \mathrm{C} ; 0,14 \mathrm{~m} / \mathrm{s} ; \operatorname{sim} . \quad---70{ }^{\circ} \mathrm{C} ; 0,42 \mathrm{~m} / \mathrm{s} ; \operatorname{sim} \text {. }
\end{aligned}
$$

Figura 4. Curvas de secagem para banana cv. D’água no formato de cilindro em diferentes condições de secagem (exp: experimentais, sim: simulados).

As Figuras 7 e 8 apresentam cinéticas de secagem para a variedade prata cortada em formato cilíndrico, com e sem o branqueamento. A Figura 7 é relativa a experimentos conduzidos a $50{ }^{\circ} \mathrm{C}$ e a Figura 8 a $70{ }^{\circ} \mathrm{C}$. Observa-se que o branqueamento sempre conduz a menores taxas de secagem. Porém, de maneira contrária ao observado para o formato disco (Figuras 5 e 6 ), sua influência é mais acentuada a menores velocidades do ar, em ambas as temperaturas tratadas.

As Figuras 9 e 10 apresentam curvas de cinética de secagem para a variedade d'água cortada em discos, com e

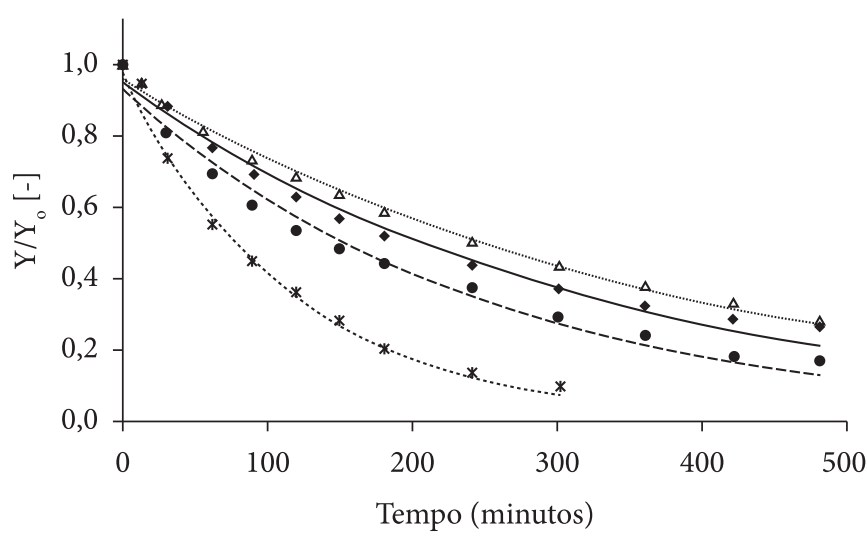

$$
\begin{aligned}
& \text { - } 0,14 \mathrm{~m} / \mathrm{s} ; \mathrm{c} . b ; \exp . \quad * \quad 0,42 \mathrm{~m} / \mathrm{s} ; \mathrm{c} . \mathrm{b} ; \exp \text {. } \\
& \text { - } 0,14 \mathrm{~m} / \mathrm{s} ; \text { c.b; sim. } \quad .-\cdots . .0,42 \mathrm{~m} / \mathrm{s} ; \text { c.b; sim. } \\
& \Delta \quad 0,14 \mathrm{~m} / \mathrm{s} ; \mathrm{s} . \mathrm{b} ; \exp . \quad-0,42 \mathrm{~m} / \mathrm{s} ; \mathrm{s} . \mathrm{b} ; \exp \text {. } \\
& \text { ……. } 0,14 \mathrm{~m} / \mathrm{s} ; \mathrm{s.b} ; \mathrm{sim} \text {. } \quad \text {--- } 0,42 \mathrm{~m} / \mathrm{s} ; \mathrm{s.b} \text {; sim. }
\end{aligned}
$$

Figura 5. Curvas de secagem para banana cv. Prata no formato de disco, $50{ }^{\circ} \mathrm{C}$ (exp: experimentais, sim: simulados, c.b: com branqueamento, s.b: sem branqueamento).

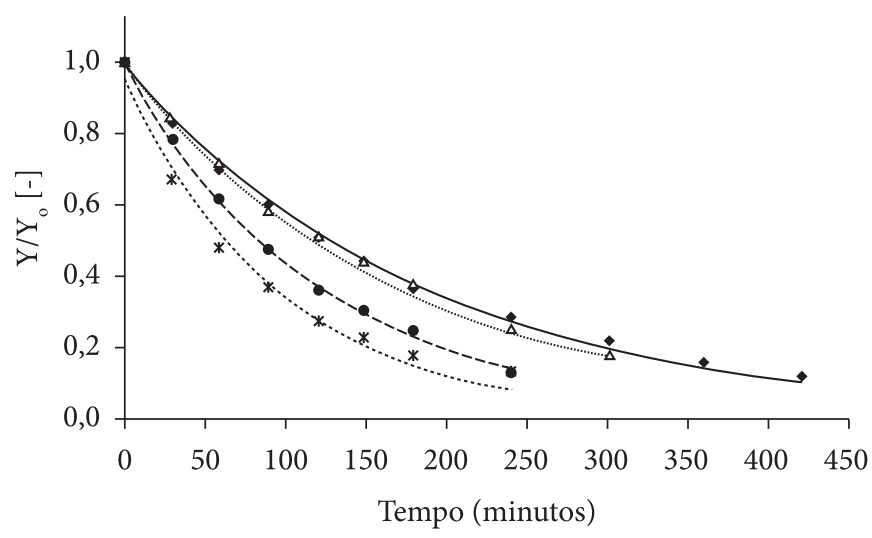

$$
\begin{aligned}
& \text { - } 0,14 \mathrm{~m} / \mathrm{s} ; \mathrm{c} . \mathrm{b} ; \exp . \quad * \quad 0,42 \mathrm{~m} / \mathrm{s} ; \mathrm{c} . \mathrm{b} ; \exp \text {. } \\
& \text { — } 0,14 \mathrm{~m} / \mathrm{s} ; \text { c.b; sim. } \quad \cdots . . . \quad 0,42 \mathrm{~m} / \mathrm{s} ; \mathrm{c} . \mathrm{b} ; \mathrm{sim} \text {. } \\
& \Delta \quad 0,14 \mathrm{~m} / \mathrm{s} ; \mathrm{s} . \mathrm{b} ; \exp . \quad \bullet \quad 0,42 \mathrm{~m} / \mathrm{s} ; \mathrm{s} . \mathrm{b} ; \exp \text {. } \\
& \text { …... } 0,14 \mathrm{~m} / \mathrm{s} ; \mathrm{s.b} ; \mathrm{sim} \text {. } \quad \text {--- } 0,42 \mathrm{~m} / \mathrm{s} ; \mathrm{s.b} \text {; sim. }
\end{aligned}
$$

Figura 6. Curvas de secagem para banana cv. Prata no formato de disco a $70^{\circ} \mathrm{C}$ (exp: experimentais, sim: simulados, c.b: com branqueamento, s.b: sem branqueamento).

sem o branqueamento, respectivamente. Observa-se que o branqueamento conduziu a uma menor taxa de secagem, sendo sua influência notável nas menores velocidades do ar. Em um trabalho de análise de pré-tratamentos para a secagem de banana, Dandamrongrak, Young e Mason (2002) compararam aumento de taxa de secagem com um congelamento prévio, branqueamento e a combinação dos dois pré-tratamentos e não observaram alteração significante da taxa de secagem com o branqueamento. Conforme já comentado em parágrafo anterior, este efeito adverso do branqueamento se deve à gelatinização do amido em temperaturas superiores a $70^{\circ} \mathrm{C}$. 


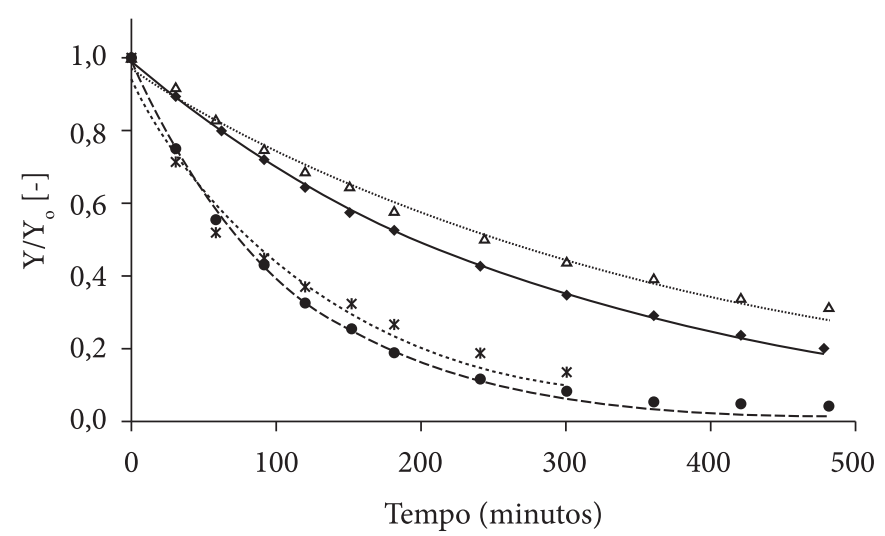

$$
\begin{aligned}
& \text { - } 0,14 \mathrm{~m} / \mathrm{s} ; \mathrm{c} . \mathrm{b} ; \exp . \quad * 0,42 \mathrm{~m} / \mathrm{s} ; \mathrm{c} . \mathrm{b} ; \exp \text {. } \\
& \text { - } 0,14 \mathrm{~m} / \mathrm{s} ; \mathrm{c} . \mathrm{b} ; \mathrm{sim} . \quad \cdots . .0,42 \mathrm{~m} / \mathrm{s} ; \text { c.b; } \operatorname{sim} \text {. } \\
& \Delta \quad 0,14 \mathrm{~m} / \mathrm{s} ; \mathrm{s} . \mathrm{b} ; \text { exp. } \quad \text { - } 0,42 \mathrm{~m} / \mathrm{s} ; \mathrm{s.b} \text {; exp. } \\
& \text {.......... } 0,14 \mathrm{~m} / \mathrm{s} ; \mathrm{s.b} \text {; sim. } \quad \text {--- } 0,42 \mathrm{~m} / \mathrm{s} \text {; s.b; sim. }
\end{aligned}
$$

Figura 7. Curvas de secagem para banana cv. Prata no formato de cilindro a $50{ }^{\circ} \mathrm{C}$ (exp: experimentais, sim: simulados, c.b: com branqueamento, s.b: sem branqueamento).

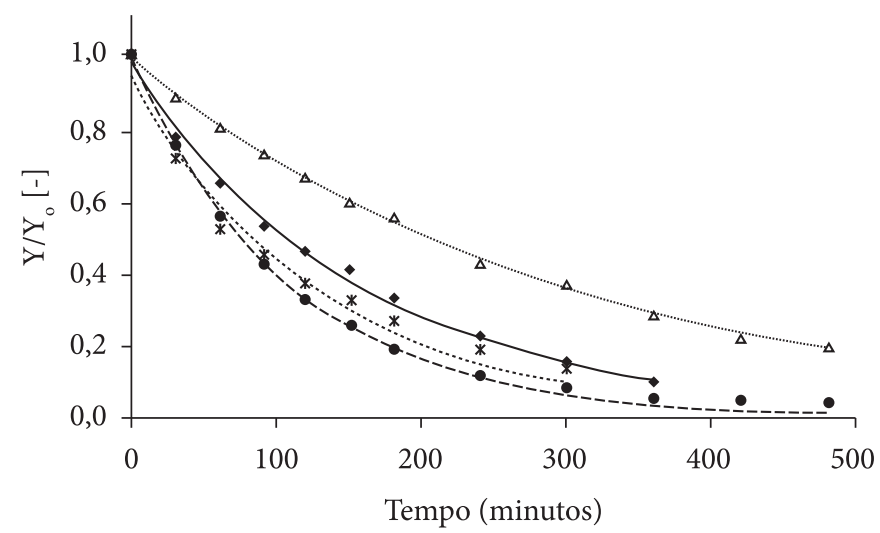

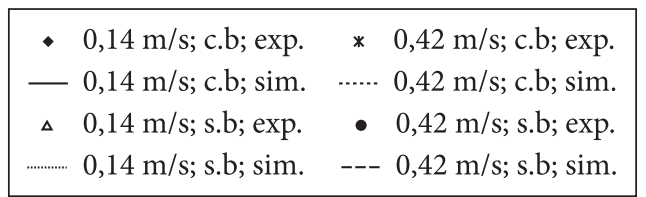

Figura 8. Curvas de secagem para banana cv. Prata cortada no formato de cilindro a $70{ }^{\circ} \mathrm{C}$ (exp: experimentais, sim: simulados, c.b: com branqueamento, s.b: sem branqueamento).

Comportamento semelhante foi observado para a cinética de secagem da banana-d'água no formato cilíndrico (Figuras 11 e 12). A $50^{\circ} \mathrm{C}$ o branqueamento não foi significante a baixas vazões de ar e diminuiu a taxa de secagem para a maior vazão de ar; a $70^{\circ} \mathrm{C}$, apenas teve um pequeno efeito na maior vazão estudada.

A influência do formato na secagem de bananas não branqueadas é apresentada nas Figuras 13 a 16. Observa-se que, somente para a variedade prata à velocidade de $0,14 \mathrm{~m} / \mathrm{s}$,

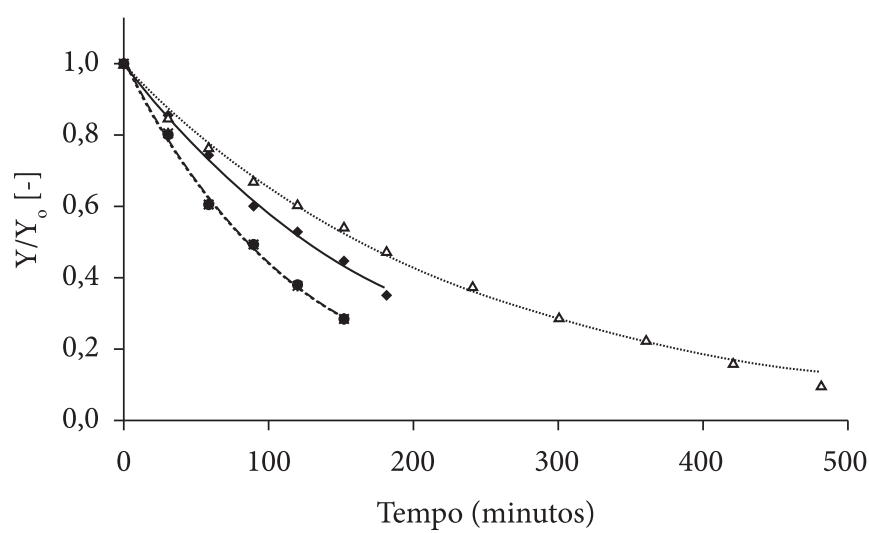

$$
\begin{aligned}
& \text { - } 0,14 \mathrm{~m} / \mathrm{s} ; \mathrm{c} . b ; \text { exp. } \quad * \quad 0,42 \mathrm{~m} / \mathrm{s} ; \mathrm{c} . \mathrm{b} ; \exp \text {. } \\
& \text { - } 0,14 \mathrm{~m} / \mathrm{s} ; \mathrm{c} . \mathrm{b} ; \mathrm{sim} . \quad \ldots . . .0,42 \mathrm{~m} / \mathrm{s} ; \mathrm{c} . \mathrm{b} ; \mathrm{sim} \text {. } \\
& \Delta \quad 0,14 \mathrm{~m} / \mathrm{s} ; \mathrm{s} . b ; \text { exp. } \quad 0,42 \mathrm{~m} / \mathrm{s} ; \mathrm{s} . b ; \text { exp. } \\
& \text { ……. } 0,14 \mathrm{~m} / \mathrm{s} \text {; s.b; sim. } \quad \text {--- } 0,42 \mathrm{~m} / \mathrm{s} \text {; s.b; sim. }
\end{aligned}
$$

Figura 9. Curvas de secagem para banana cv. D'água no formato de disco a $50{ }^{\circ} \mathrm{C}$ (exp: experimentais, sim: simulados, c.b: com branqueamento, s.b: sem branqueamento).

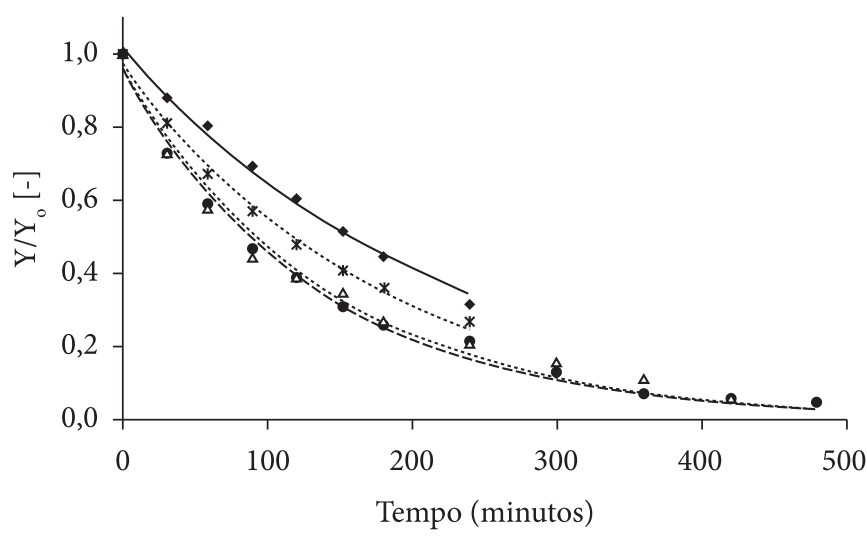

$$
\begin{aligned}
& \text { - } 0,14 \mathrm{~m} / \mathrm{s} ; \text { c.b; exp. } \quad * 0,42 \mathrm{~m} / \mathrm{s} ; \text { c.b; exp. } \\
& \text { - } 0,14 \mathrm{~m} / \mathrm{s} ; \text { c.b; sim. } \quad \cdots \cdots . . .0,42 \mathrm{~m} / \mathrm{s} ; \text { c.b; sim. } \\
& \Delta \quad 0,14 \mathrm{~m} / \mathrm{s} ; \mathrm{s} . \mathrm{b} ; \exp . \quad-0,42 \mathrm{~m} / \mathrm{s} ; \mathrm{s} . \mathrm{b} ; \exp \text {. } \\
& \text { ….... } 0,14 \mathrm{~m} / \mathrm{s} ; \mathrm{s} . \mathrm{b} ; \mathrm{sim} . \quad \text {--- } 0,42 \mathrm{~m} / \mathrm{s} \text {; s.b; sim. }
\end{aligned}
$$

Figura 10. Curvas de secagem para banana cv. D'água no formato de disco a $70{ }^{\circ} \mathrm{C}$ (exp: experimentais, sim: simulados, c.b: com branqueamento, s.b: sem branqueamento).

o formato foi influente, sendo que o formato disco resultou em maiores taxas de secagem nas duas temperaturas analisadas. Menores velocidades de ar significam maiores resistências externas à secagem e, desta forma, um formato que proporcione maior superfície de contato tende a fornecer maiores taxas de secagem, conforme o observado na Figura 13.

As Figuras 17 e 18 apresentam comparações entre as duas cultivares de bananas sem branqueamento, a $0,14 \mathrm{~m} / \mathrm{s}$, nos formatos disco e cilíndrico, respectivamente. Observa-se que, 
para o formato disco, a variedade somente foi influente a $70^{\circ} \mathrm{C}$, temperatura em que a taxa de secagem foi maior para bananad'água. Para o formato cilíndrico, somente a variedade d’água a $70{ }^{\circ} \mathrm{C}$ apresentou variações significativas das demais situações estudadas, apresentando maior taxa de secagem. Ao se analisar as Figuras 17 e 18, pode-se dizer que a temperatura foi mais influente que a variedade.
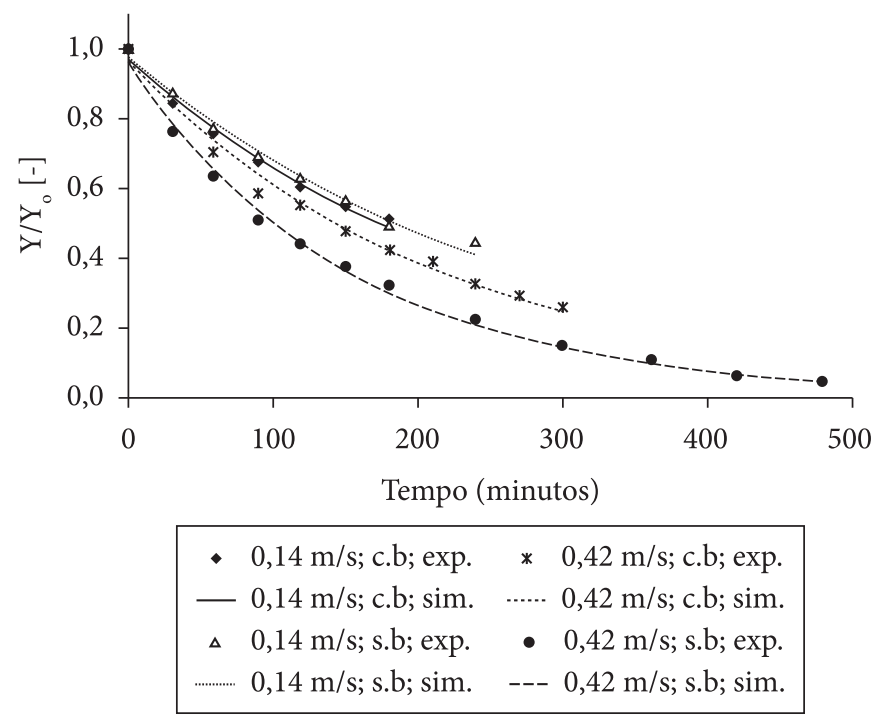

Figura 11. Curvas de secagem para banana cv. D'água no formato de cilindro a $50{ }^{\circ} \mathrm{C}$ (exp: experimentais, sim: simulados, c.b: com branqueamento, s.b: sem branqueamento).
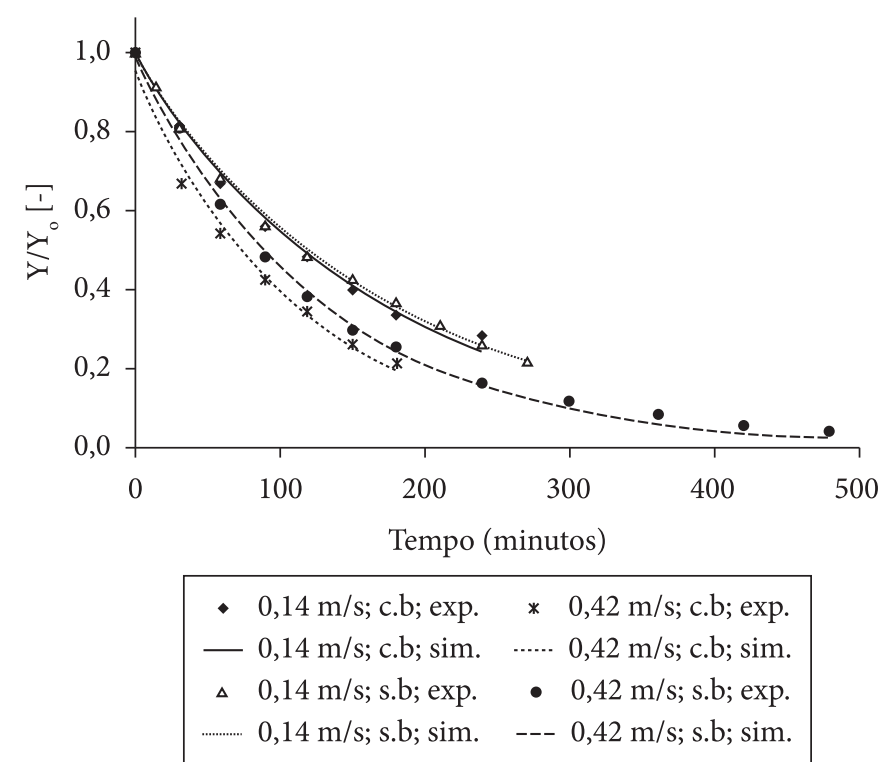

Figura 12. Curvas de secagem para banana cv. D'água no formato de cilindro a $70{ }^{\circ} \mathrm{C}$ (exp: experimentais, sim: simulados, c.b: com branqueamento, s.b: sem branqueamento).

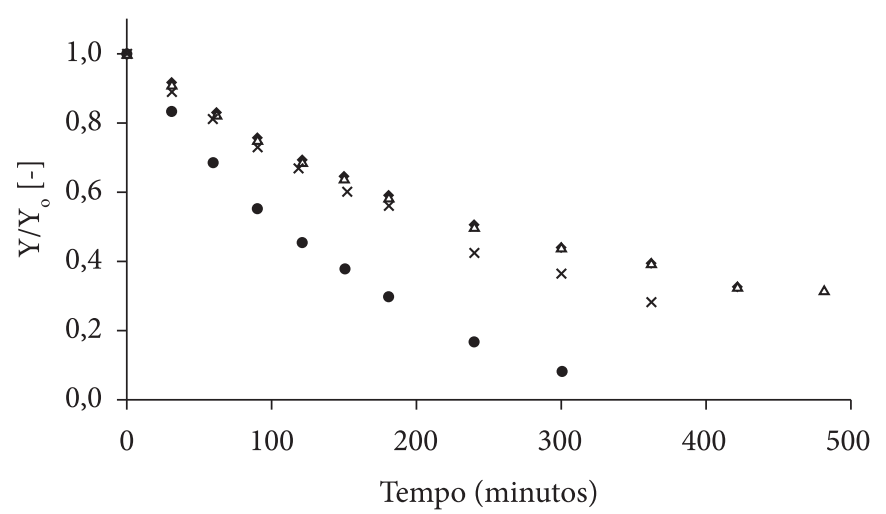

- Disco; $50^{\circ} \mathrm{C} \quad{ }_{\Delta}$ Cilindro; $50^{\circ} \mathrm{C}$

- Disco; $70{ }^{\circ} \mathrm{C} \quad \times$ Cilindro; $70^{\circ} \mathrm{C}$

Figura 13. Curvas de secagem para banana cv. Prata sem branqueamento a $0,14 \mathrm{~m} / \mathrm{s}$.

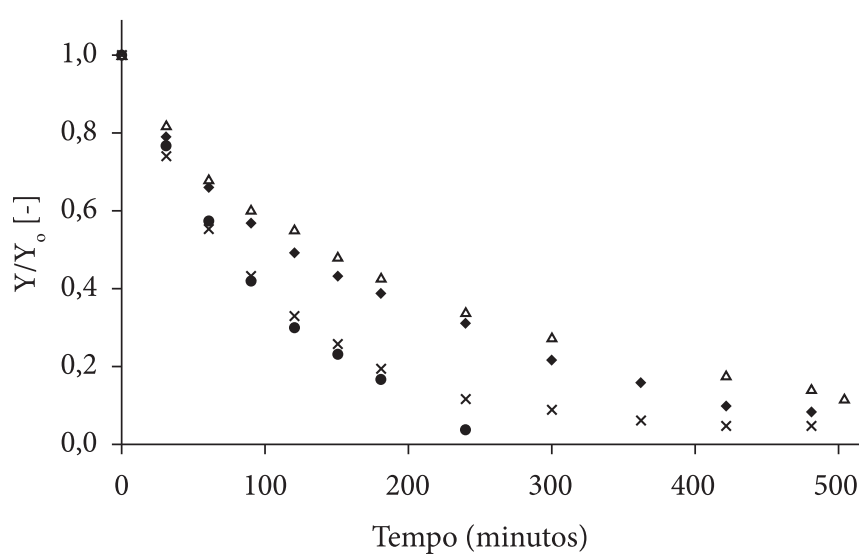

- Disco; $50^{\circ} \mathrm{C} \quad \triangle$ Cilindro; $50^{\circ} \mathrm{C}$

- Disco; $70^{\circ} \mathrm{C} \times$ Cilindro; $70^{\circ} \mathrm{C}$

Figura 14. Curvas de secagem para banana variedade prata sem branqueamento a $0,42 \mathrm{~m} / \mathrm{s}$.

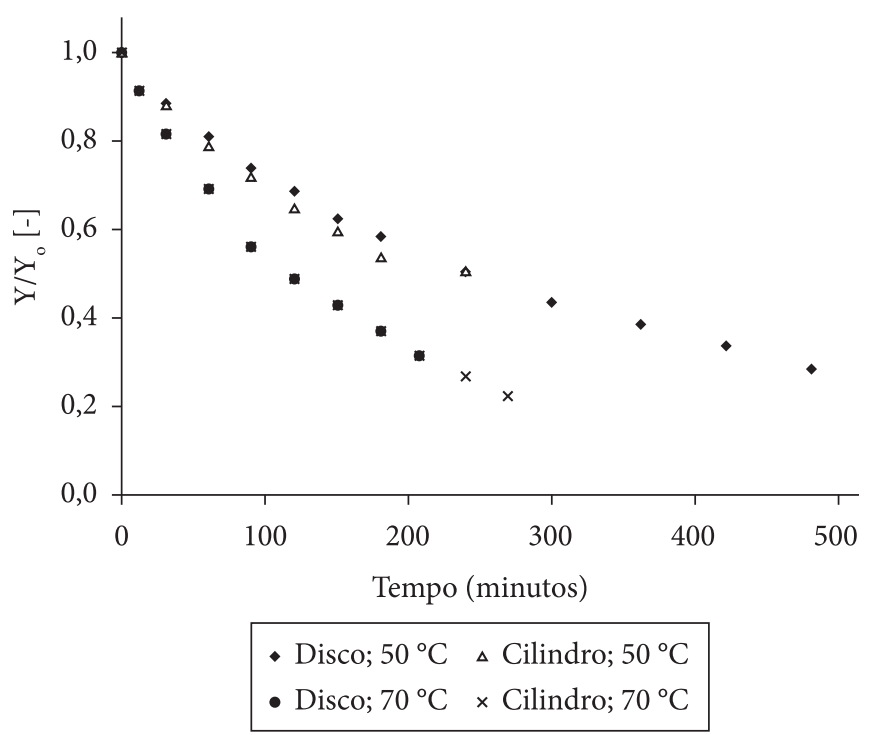

Figura 15. Curvas de secagem para banana cv. D'água sem branqueamento a $0,14 \mathrm{~m} / \mathrm{s}$. 


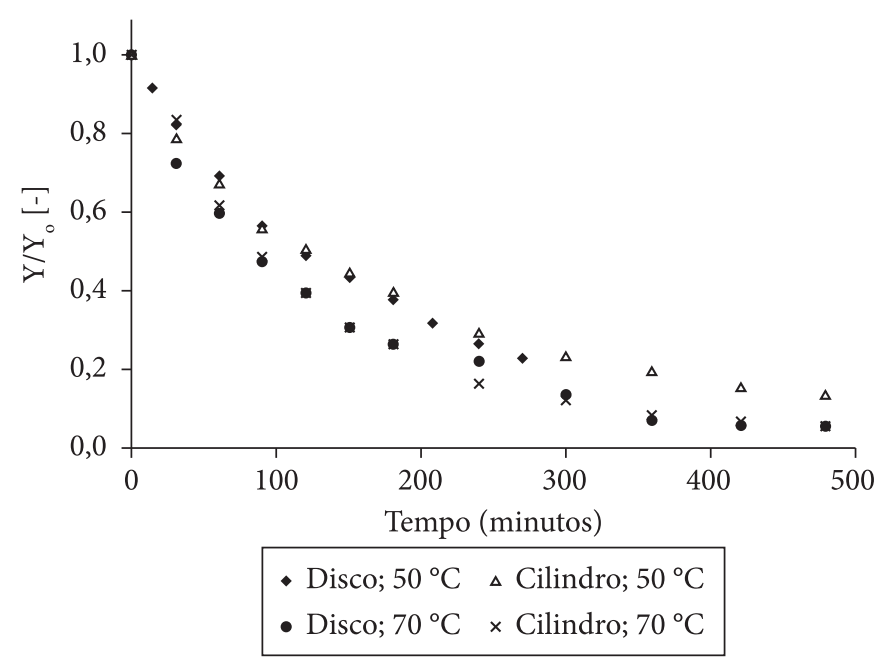

Figura 16. Curvas de secagem para banana cv. D’água sem branqueamento a $0,42 \mathrm{~m} / \mathrm{s}$.

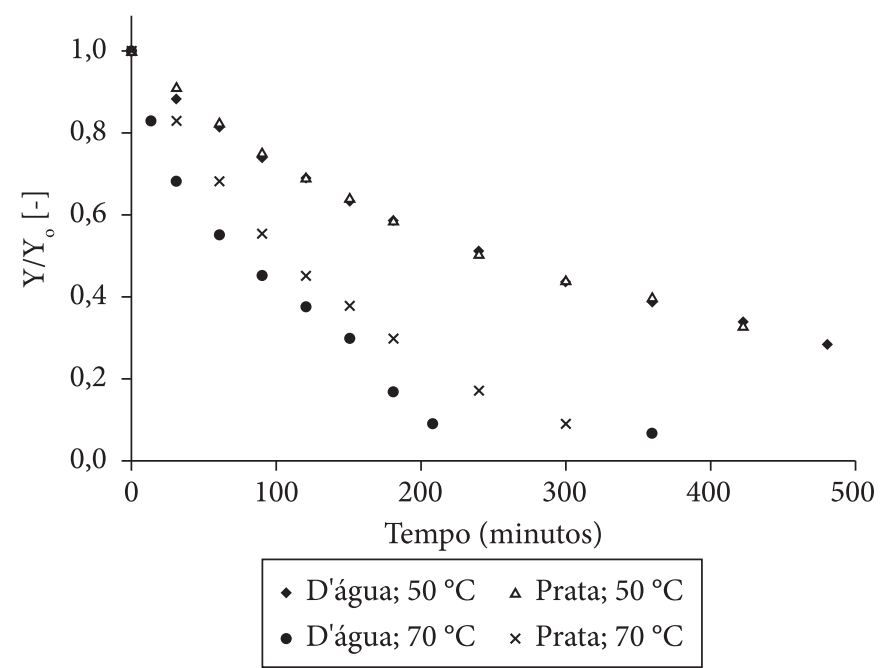

Figura 17. Curvas de secagem de banana sem branqueamento no formato de disco a $0,14 \mathrm{~m} / \mathrm{s}$.

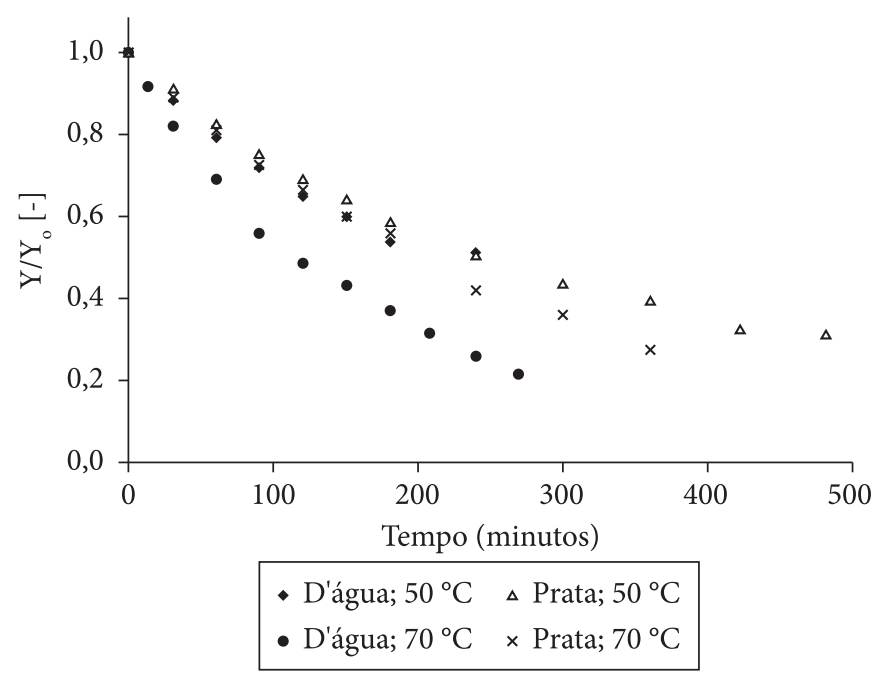

Figura 18. Curvas de secagem para banana sem branqueamento no formato de cilindro a $0,14 \mathrm{~m} / \mathrm{s}$.

\section{Conclusões}

$\mathrm{O}$ modelo exponencial foi bem ajustado às curvas de secagem $\left(R^{2}: 0,98-0,99\right.$, e baixos valores de SE).

De acordo com as constantes cinéticas apresentadas pelo modelo, recomenda-se a secagem de bananas em quaisquer formatos nas seguintes condições: branqueamento e secagem a $50{ }^{\circ} \mathrm{C} / 0,42 \mathrm{~m} / \mathrm{s}$, para banana-prata; e secagem a $70{ }^{\circ} \mathrm{C} / 0,42 \mathrm{~m} / \mathrm{s}$, para banana-d’água sem branqueamento.

\section{Referências bibliográficas}

ALCINA, O. L. S.; SILVA, O. S.; BRASILEIRO, M. N. Drying kinetics of West Indian Cherry. In: INTER-AMERICAN DRYING CONFERENCE, 1997, Itu. Proceedings... Itu: IADC, 1997. p. 434-440.

AREVALO-PINEDO, A.; MURR, F. E. Influence of pre-treatments on the drying kinetics during vacuum drying of carrot and pumpkin. Journal of Food Engineering, v. 80, n. 1, p. 152-156, 2007.

ASSOCIATION OF OFFICIAL ANALYTICAL CHEMISTS - AOAC. Official Methods of Analysis. 17 ed. Maryland, 2002.

BITTENCOURT, J.; QUEIROZ, M. R.; NEBRA, S. A. Avaliação econômica da elaboração de banana-passa proveniente do cultivo orgânico e convencional. Engenharia Agrícola, v. 24, n. 2, p. 473-483, 2004.

BOUDHRIOUA, N.; GIAMPAOLI, P.; BONAZZI, C. Changes in aromatic components of banana during ripening and air-drying Lebensmittel Wissenschaft und Technology, v. 36, p. 633-642, 2003.

CANO-CHAUCA, M. et al. Drying curves and water evaluation of dryed banana. In: INTERNATIONAL DRYING SYMPOSIUM, 14, 2004, São Paulo. Proceedings... São Paulo: IDS, 2004. p. 2013-2020.

CORRÊA, J. L. G. et al. Analysis of osmotic dehydration variables: Influences on tomato (Licopersicon esculentum) drying. Boletim do Centro de Pesquisa e Processamento de Alimentos, v. 25, n. 2, p. 315-328, 2007.

CORRÊA, J. L. G. et al. Desidratação osmótica de tomate seguida de secagem. Revista Brasileira de Produtos Agroindustriais, v. 10, n. 1, p. 35-42, 2008.

DANDAMRONGRAK, R.; YOUNG, G.; MASON, R. Evaluation of various pre-treatments for the dehydration of banana and selection of suitable drying models. Journal of Food Engineering, v. 55, p. 139-146, 2002

DEMIREL, D.; TURHAN, M. Air-drying behavior of Dwarf Cavendish and Gros Michel banana slices. Journal of Food Engineering, v. 59, n. 1, p. 1-11, 2003.

FERNANDES, F. A. N. et al. Optimization of osmotic dehydration of bananas followed by air-drying. Journal of Food Engineering, v. 77, n. 1, p. 188-193, 2006.

GIRALDO-ZUNIGA, A. et al. Drying kinetics for murici (Byrsonima crassifolia) Fruit. Journal of Food Processing and Preservation, v. 30, p. 699-705, 2006.

INSTITUTO ADOLFO LUTZ - IAL. Normas analíticas do Instituto Adolfo Lutz - métodos químicos e físicos para análise de alimentos. São Paulo, 1985.

INSTITUTO BRASILEIRO DE GEOGRAFÍA E ESTATÍSTICA IBGE. Previsão de safra-Brasil 2006-2007. Disponível em: www:sidra.ibge.gov.br. Acesso em: 04 dez. 2007. 
KATEKAWA, M. E.; SILVA, M. A. On the influence of glass transition on shrinkage in convective drying of fruits: a case study of banana. Drying Technology, v. 25, p. 1659-1666, 2007.

KAYA, A.; AYDIN, O.; DINCER, I. Experimental and numerical investigation of heat and mass transfer during drying of Hayward kiwi fruits (Actinidia Deliciosa Planch). Journal of Food Engineering, v. 88, p. 323-330, 2008.

KROKIDA, M. K. et al. Drying Kinetics of some vegetables. Journal of Food Engineering, v. 59, n. 4, p. 391-403, 2003.

LAHSASNI, S. et al. Drying kinetics of prickly pear fruit (Opuntia ficus indica). Journal of Food Engineering, v. 61, p. 173-179, 2004.

LEITE, J. B. Uma contribuição ao estudo da secagem de banana em secadores de bandejas. $100 \mathrm{f}$. Dissertação (Mestrado em Ciências dos Alimentos) - Departamento de Engenharia Química, Universidade Federal Rural do Rio de Janeiro, Seropédica, 2002.

LEITE, J. B.; MANCINI, M. C.; BORGES, S. V. Effect of drying temperature on the quality of dried bananas cv. prata and d'água. Lebensmittel Wissenschaft und Technology, v. 40, n. 2, p. 319-323, 2007.

LEWICKI, P. P.; PAWLAK, G. Effect of drying on microstructure of plant tissue. Drying Technology, v. 21, n. 4, p. 657-683, 2003.
MADAMBA, P. S.; DRISCOLL, R. H.; BUCLE, K. A. The thinlayer drying characateristicas of garlic slices. Journal of Food Engineering, v. 29, n. 1, p. 75-97, 1996.

MIDILLI, A.; KUCUK, H.; YAPAR, Z. A new model for single-layer drying. Drying Technology, v. 20, n. 7, p. 1503-1513, 2002.

MOTA, R. V. Avaliação da qualidade de banana passa elaborada a partir de 6 cultivares. Ciência e Tecnologia de Alimentos, v. 25, n. 3, p. 560-563, 2005.

NAGLE, M. et al. Effects of operating practices on performance of a fixed-bed convection dryer and quality of dried longan. International Journal of Food Science and Technology, v. 43, p. 1979-1987, 2008.

PIMENTEL-GOMES, F. Curso de estatística experimental. São Paulo: Nobel, 1999.

QUEIROZ, M. R.; NEBRA, S. A. Theoretical and experimental analysis of drying kinetics of bananas. Journal of Food Engineering, v. 47, n. 2, p. 127-132, 2001.

TRAVAGLINI, D. A. et al. Banana-passa-princípios de secagem. Campinas: ITAL, 1993. (Manual Técnico no. 12). 